\title{
Sheaf Toposes for Realizability
}

\author{
Steven Awodey* \\ Philosophy Department \\ Carnegie Mellon University \\ Andrej Bauer ${ }^{\dagger}$ \\ Department of Mathematics and Physics \\ University of Ljubljana
}

September 7, 2004

\begin{abstract}
We compare realizability models over partial combinatory algebras by embedding them into sheaf toposes. We then use the machinery of Grothendieck toposes and geometric morphisms to study the relationship between realizability models over different partial combinatory algebras. This research is part of the Logic of Types and Computation project at Carnegie Mellon University under the direction of Dana Scott.
\end{abstract}

\section{Introduction}

The purpose of this paper is to compare realizability models over partial combinatory algebras by embedding them into sheaf toposes. We then use the machinery of Grothendieck toposes and geometric morphisms to study the relationship between realizability models over different partial combinatory algebras. This work is related to Rosolini and Streicher [RS99], where the focus was mainly on the locally cartesian closed structure of realizability models, as well as to Mulry [Mul82] and Rosolini [Ros86]. Here we are also interested in comparison of logical properties.

As a reference on topos theory we use [MM92], and Johnstone and Moerdijk [JM89] as a reference on local map of toposes. Longley's dissertation [Lon94] contains material on partial combinatory algebras, applicative morphisms, and realizability. In Birkedal's dissertation [Bir00] and in [ABS99] you can find further information about realizability, and also on local maps of toposes and the corresponding $\sharp-b$ calculus.

${ }^{*} 5000$ Forbes Ave., Pittsburgh, PA 15213, USA; E-mail: awodey@andrew.cmu.edu

${ }^{\dagger}$ Jadranska 19, 1000 Ljubljana, Slovenia. E-mail: Andrej .Bauer@andrej.com 
Acknowledgement. We thank Peter Johnstone, Bill Lawvere, and Alex Simpson for helpful discussions.

\section{Sheaves on Partial Combinatory Algebras}

We quickly review the basic definitions about partial combinatory algebras and applicative morphisms. For motivation and examples of PCAs and applicative morphisms, see [Lon94]. For a partial function $f: A \rightarrow B$ and $x \in A$, the notation $f x \downarrow$ means that $f x$ is defined. When $t$ is a term, $t \downarrow$ means that the value of $t$, and hence of all of its subterms, is defined.

Definition 2.1 A partial combinatory algebra $(P C A)$ is a set $\mathbb{A}$ with a partial application operation $\square \cdot \square: \mathbb{A} \times \mathbb{A} \rightarrow \mathbb{A}$ and distinguished elements $\mathrm{K}, \mathrm{S} \in \mathbb{A}$ such that, for all $x, y, z \in \mathbb{A}$,

$$
\mathrm{K} x y \simeq x, \quad \mathrm{~S} x y \downarrow, \quad \mathrm{S} x y z \simeq(x z)(y z),
$$

where $\simeq$ means that if one side is defined then so it the other and they are equal. We usually write $x y$ instead of $x \cdot y$, and associate application to the left. Here we only consider non-trivial PCAs that satisfy $\mathrm{K} \neq \mathrm{S}$.

Example 2.2 Scott's graph model $\mathbb{P}=\mathcal{P N}$, [Sco76], is a model of the untyped $\lambda$-calculus, therefore a (total) combinatory algebra. The algebraic lattice of continuous maps $\mathbb{P}^{\mathbb{P}}$ is a retract of the algebraic lattice $\mathbb{P}$. The embedding $\Gamma: \mathbb{P}^{\mathbb{P}} \rightarrow \mathbb{P}$ is defined by

$$
\Gamma f=\{\langle m, n\rangle \mid n \in \mathbb{N} \text { and } m \in f(\text { finset } n)\},
$$

where $\langle m, n\rangle$ is an effective pairing of natural numbers and finset $n$ is an effective enumeration of finite subsets of $\mathbb{N}$. The set $\Gamma f$ is called the graph of $f$. For a continuous map $f: \mathbb{P} \rightarrow \mathbb{P}$ the graph $\Gamma f$ uniquely determines $f$ because the value of $f$ at any element $x \in \mathbb{P}$ is the union of values of $f$ at finite subsets of $x$. The retraction $\Lambda: \mathbb{P} \rightarrow \mathbb{P}^{\mathbb{P}}$ is defined by

$$
(\Lambda x) y=\{m \in \mathbb{N} \mid \exists n \in \mathbb{N} .(\langle m, n\rangle \in x \text { and finset } n \subseteq y)\} .
$$

Define application on $\mathbb{P}$ by

$$
x \cdot y=(\Lambda x) y,
$$

and $\lambda$-abstraction by

$$
\lambda u . \phi(u)=\Gamma(\lambda x: \mathbb{P} \cdot \phi(x)) .
$$

Here $\phi(u)$ is an expression with $u$ occurring as a free variable, and involving further $\lambda$-abstractions and applications of elements of $\mathbb{P}$. By taking $\mathrm{K}=$ $\lambda x y . x$ and $\mathbf{S}=\lambda x y z .(x z)(y z), \mathbb{P}$ becomes a total combinatory algebra for the above application operation. 
Example 2.3 The first Kleene Algebra $\mathbb{K}$ is the set of natural numbers $\mathbb{N}$ equipped with Kleene application $n \cdot m=\{n\} m$ which applies the $n$-th partial recursive function $\{n\}$ to $m$. The existence of $\mathbf{K}$ and $\mathbf{S}$ is a consequence of the s-m-n theorem [Rog87].

Example 2.4 The second Kleene Algebra $\mathbb{B}=\mathbb{N}^{\mathbb{N}}$, or the Baire space, is a partial combinatory algebra. The continuous partial application is defined as follows. If $\alpha \in \mathbb{N}^{\mathbb{N}}$, let $\bar{\alpha} n$ be the sequence $[\alpha 0, \ldots, \alpha(n-1)]$, encoded reasonably as a natural number. Define the operation $\star$ by

$$
\alpha \star \beta=n \Longleftrightarrow \exists m \in \mathbb{N} .(\alpha(\bar{\beta} m)=n+1 \wedge \forall k<m . \alpha(\bar{\beta} k)=0) .
$$

Now the partial application on $\mathbb{B}$ is defined by

$$
(\alpha \mid \beta) n=\alpha \star(n:: \beta),
$$

where $n:: \beta$ is the sequence obtained by prepending $n$ to $\beta$. We consider $\alpha \mid \beta$ to be defined only if $(\alpha \mid \beta) n$ is defined for all $n \in \mathbb{N}$. For details about the PCA structure of $\mathbb{B}$, see [KV65].

Definition 2.5 (John Longley) Let $\mathbb{E}$ and $\mathbb{F}$ be PCAs. An applicative morphism $\rho: \mathbb{E} \stackrel{\mathrm{PCA}}{\longrightarrow} \mathbb{F}$ is a total relation $\rho \subseteq \mathbb{E} \times \mathbb{F}$ for which there exists $r \in \mathbb{F}$, such that $r x \downarrow$ for all $x \in \mathbb{F}$, and for all $u, v \in \mathbb{E}, x, y \in \mathbb{F}$, if $\rho(u, x)$, $\rho(v, y)$ and $u v \downarrow$, then $r x y \downarrow$ and $\rho(u v, r x y)$. We say that $r$ is a realizer for the applicative morphism $\rho$. The composition of applicative morphisms is the usual composition of relations. An applicative morphism is discrete when $\rho(u, x)$ and $\rho(v, x)$ implies $u=v$.

Suppose $\rho, \sigma: \mathbb{E} \stackrel{\text { PCA }}{\longrightarrow} \mathbb{F}$ are applicative morphisms. We say there is an applicative transformation from $\rho$ to $\sigma$, and write $\rho \preceq \sigma$, if there exists $t \in \mathbb{F}$ such that whenever $\rho(u, x)$ then $\sigma(u, t x)$. When $\rho \preceq \sigma$ and $\sigma \preceq \rho$ we write $\rho \sim \sigma$.

Example 2.6 A discrete applicative morphism $\iota: \mathbb{B} \stackrel{\text { PCA }}{\longrightarrow} \mathbb{P}$ can be obtained by embedding $\mathbb{B}$ into $\mathbb{P}$ via $\iota a=\{\bar{a} n \mid n \in \mathbb{N}\}$, where $\bar{a} n \in \mathbb{N}$ is the sequence $[a 0, \ldots, a(n-1)]$ suitably encoded as a natural number. Note that $\iota$ is actually a function, i.e., a single-valued total relation. There is also a discrete applicative morphism $\delta: \mathbb{P} \stackrel{\text { PCA }}{\longrightarrow} \mathbb{B}$, defined by

$$
\delta(x, a) \Longleftrightarrow x=\{n \in \mathbb{N} \mid \exists k \in \mathbb{N} . a k=n+1\} .
$$

In words, a sequence $a$ is a $\delta$-implementation of $x$ if it enumerates the elements of $x$. The trick with adding 1 ensures that the empty set is accounted for.

Applicative morphisms induce functors between realizability toposes. We only consider discrete applicative morphisms here, because they induce functors between categories of modest sets. 
Definition 2.7 Let $\mathbb{A}$ be a PCA. A modest set $\left(S, \Vdash_{S}\right)$ over $\mathbb{A}$ is a set $S$ with a realizability relation $\Vdash_{S} \subseteq \mathbb{A} \times S$ such that for every $x \in S$ there exists $a \in \mathbb{A}$ such that $a \Vdash_{S} x$, and for all $a \in \mathbb{A}, x, y \in S$,

$$
\left(a \Vdash_{S} x\right) \wedge\left(a \Vdash_{S} y\right) \Longrightarrow x=y .
$$

A realized function $f:\left(S, \Vdash_{S}\right) \rightarrow\left(T, \Vdash_{T}\right)$ between modest sets is a function $f: S \rightarrow T$ that is tracked by some $a \in \mathbb{A}$, which means that, for all $x \in S$,

$$
\left(b \Vdash_{S} x\right) \Longrightarrow a b \downarrow \wedge\left(a b \Vdash_{T} f x\right) .
$$

The category of modest sets and realized functions is denoted by $\operatorname{Mod}(\mathbb{A})$.

The category of modest sets $\operatorname{Mod}(\mathbb{A})$ is equivalent to the perhaps better known category $\operatorname{PER}(\mathbb{A})$ of partial equivalence relations on $\mathbb{A}$.

Example 2.8 The category $\operatorname{Mod}(\mathbb{P})$ is equivalent to the category of countably based equilogical spaces [BBS98]. A countably based equilogical space is a pair $\left(X, \equiv_{X}\right)$ where $X$ is a countably based $T_{0}$-space and $\equiv$ is an equivalence relation on $X$. A morphism $[f]:\left(X, \equiv_{X}\right) \rightarrow\left(Y, \equiv_{Y}\right)$ is an equivalence class of equivalence preserving continuous maps. Two such maps are considered equivalent when they map equivalent points to equivalent points.

Example 2.9 The category $\operatorname{Mod}(\mathbb{K})$ is closely related to Eršov's numbered set. The modest sets over $\mathbb{K}$ are equivalent to the category of partial numerings, which are partial surjections $\mathbb{N} \rightarrow S$. Eršov's numbered sets are the total surjections from $\mathbb{N}$, and in $\operatorname{Mod}(\mathbb{K})$ they are the regular quotients of the natural number object.

Example 2.10 The category $\operatorname{Mod}(\mathbb{B})$ is the ambient category of Type Two Effectivity [Wei00]. It is equivalent to 0-equilogical spaces [Bau00], which is the full subcategory on those countably based equilogical spaces whose underlying topological space is 0-dimensional.

Any discrete applicative morphism $\rho: \mathbb{E} \stackrel{\text { PCA }}{\longrightarrow} \mathbb{F}$ induces a functor

$$
\widehat{\rho}: \operatorname{Mod}(\mathbb{E}) \longrightarrow \operatorname{Mod}(\mathbb{F}),
$$

defined as follows. A modest set $\left(S, \Vdash_{S}\right)$ over $\mathbb{E}$ is mapped to $\widehat{\rho}\left(S, \Vdash_{S}\right)=$ $\left(S, \Vdash_{\widehat{\rho} S}\right)$ where $\Vdash_{\widehat{\rho} S}$ is defined by

$$
a \Vdash_{\widehat{\rho} S} x \Longleftrightarrow \exists b \in \mathbb{E} .\left(\left(b \Vdash_{S} x\right) \wedge \rho(b, a)\right) .
$$

A realized map $f:\left(S, \Vdash_{S}\right) \rightarrow\left(T, \Vdash_{T}\right)$ is mapped to the same map $\widehat{\rho} f=$ $f: S \rightarrow T$. To see that $\widehat{\rho} f$ is realized, let $r \in \mathbb{F}$ be a realizer for $\rho$, and let $a \in \mathbb{E}$ be a realizer for $f$. Because $\rho$ is total there exists $b \in \mathbb{F}$ such that $\rho(a, b)$, and $r b$ is a realizer for $\widehat{\rho} f$. 


\subsection{Sheaves over a PCA}

We would like to embed $\operatorname{Mod}(\mathbb{A})$ into a sheaf topos. An obvious choice is the topos of sheaves for a subcanonical Grothendieck topology on $\operatorname{Mod}(\mathbb{A})$, which is generated by suitable regular epimorphic families. There is an equivalent but much simpler description of this topos, which is obtained as follows.

For the site we take the category $\langle\mathbb{A}\rangle$ whose objects are subsets of $\mathbb{A}$, and morphisms are the realized maps between subsets of $\mathbb{A}$. More precisely, if $X, Y \subseteq \mathbb{A}$ then a morphism $f: X \rightarrow Y$ is a function $f: X \rightarrow Y$ for which there exists $a \in \mathbb{A}$ such that, for all $b \in X, a b \downarrow$ and $f b=a b$.

In many cases $\langle\mathbb{A}\rangle$ is equivalent to a well known category. For example, $\langle\mathbb{P}\rangle$ is equivalent to the category $\omega$ Top $_{0}$ of countably based $T_{0^{-}}$ spaces, whereas $\langle\mathbb{B}\rangle$ is equivalent to the category 0Dim of countably based 0-dimensional Hausdorff spaces.

In order to obtain a convenient description of the Grothendieck topology on $\langle\mathbb{A}\rangle$ we need to know precisely which coproducts exist in $\langle\mathbb{A}\rangle$. For this the purpose we prove Lemma 2.11 below. Let us call a family $\left(X_{i}\right)_{i \in I}$ non-trivial if none of the objects $X_{i}$ are initial. We are only interested in coproducts of non-trivial families because we can always omit any summands that are initial. The cardinality of a set $I$ is denoted by $|I|$.

Lemma 2.11 Let $\mathbb{A}$ be a $P C A$. There exists a regular cardinal number $\mathrm{w}(\mathbb{A})$, called the weight of $\mathbb{A}$, such that the coproduct of a non-trivial family $\left(X_{i}\right)_{i \in I}$ exists in $\langle\mathbb{A}\rangle$ if, and only if, $|I|<\mathrm{w}(\mathbb{A})$.

Proof. We prove the following statement: if a coproduct of a non-trivial family $\left(X_{i}\right)_{i \in I}$ exists in $\langle\mathbb{A}\rangle$, then coproducts of all $I$-indexed families exist. We then take as the weight of $\mathbb{A}$ the smallest cardinal $\kappa$ for which there exists a non-trivial $\kappa$-indexed family such that its coproduct does not exist in $\langle\mathbb{A}\rangle$.

Let $\left(X_{i}\right)_{i \in I}$ be a non-trivial family and let $C=\coprod_{i \in I} X_{i}$ be its coproduct in $\langle\mathbb{A}\rangle$, with canonical injections $e_{i}: X_{i} \rightarrow C$ that are realized by $r_{i} \in \mathbb{A}$, for each $i \in I$. We denote the image of $X_{i}$ under $e_{i}$ by $e_{i}\left(X_{i}\right)$.

First we show that the coproduct of $(1)_{i \in I}$ exists. An object in $\langle A\rangle$ is initial if, and only if, it is the empty set. Because $\left(X_{i}\right)_{i \in I}$ is non-trivial the sets $e_{i}\left(X_{i}\right)$ are non-empty, hence there exists a choice function $c: I \rightarrow \mathbb{A}$ such that $c_{i} \in e_{i}\left(X_{i}\right)$ for all $i \in I$. The function $c$ is injective because $e_{i}\left(X_{i}\right) \cap$ $e_{j}\left(X_{j}\right)=\emptyset$ when $i \neq j$. We show that $S=\left\{c_{i} \mid i \in I\right\}$ is a coproduct of the family $(1)_{i \in I}$ with the $i$-th canonical injection realized by $\mathrm{K} c_{i}$. It suffices to find a realizer for an arbitrary function $f: S \rightarrow \mathbb{A}$. Let $g_{i}: X_{i} \rightarrow \mathbb{A}$ be the morphism realized by $\mathrm{K}\left(f\left(c_{i}\right)\right)$. Then there exists a unique morphism $g: C \rightarrow \mathbb{A}$, realized by some $s \in \mathbb{A}$, such that $g\left(e_{i}(x)\right)=g_{i}(x)=f\left(c_{i}\right)$ for all $i \in I$ and all $x \in X_{i}$. In particular, $f\left(c_{i}\right)=g\left(c_{i}\right)=s c_{i}$ for all $i \in I$, hence $s$ realizes $f$. 
Now let $\left(Y_{i}\right)_{i \in I}$ be an arbitrary $I$-indexed family. We claim that the set $T=\left\{\left\langle c_{i}, u\right\rangle \mid i \in I \wedge u \in Y_{i}\right\}$ is a coproduct of $\left(Y_{i}\right)_{i \in I}$ with the $i$-th canonical injection realized by $\lambda^{*} u .\left\langle c_{i}, u\right\rangle$. Let $\left(g_{i}: Y_{i} \rightarrow Z\right)_{i \in I}$ be a cocone, where $g_{i}$ is realized by $t_{i} \in \mathbb{A}$ for every $i \in I$. We only need to show that the map $\left\langle c_{i}, u\right\rangle \mapsto g_{i}(u): T \rightarrow Z$ is realized. Define $f: S \rightarrow \mathbb{A}$ by $f\left(c_{i}\right)=t_{i}$. Because $S$ is a coproduct of 1 's $f$ is realized by some $s \in \mathbb{A}$. The map $\left\langle c_{i}, u\right\rangle \mapsto g_{i}(u)$ is then realized by $\lambda^{*} w$. $(s($ fst $w))$ (snd $\left.w\right)$.

It remains to show that $w(\mathbb{A})$ is a regular cardinal. It is necessarily infinite because $\langle\mathbb{A}\rangle$ always has all finite coproducts. By [Jec97, Lemma 3.6], it suffices to show that $\sum_{i<\lambda} \kappa_{i}<\mathrm{w}(\mathbb{A})$ whenever $\lambda<\mathrm{w}(\mathbb{A})$ and $\kappa_{i}<\mathrm{w}(\mathbb{A})$ for all $i<\lambda$. For every $i<\lambda$, the coproduct $K_{i}=\coprod_{j<\kappa_{i}} 1$ exists in $\langle\mathbb{A}\rangle$ because $\kappa_{i}<\mathrm{w}(\mathbb{A})$. Similarly, the coproduct $L=\coprod_{i<\lambda} K_{i}$ exists. But $L$ is also a coproduct of $\sum_{i<\lambda} \kappa_{i}$ many copies of 1 , therefore $\sum_{i<\lambda} \kappa_{i}<\mathrm{w}(\mathbb{A})$.

The name weight of a PCA is motivated by the fact that in a topological PCA it often happens that its weight is the successor of its topological weight, which is the cardinality of a minimal base for its topology. For example, the topological weights of $\mathbb{P}$ and $\mathbb{B}$ are $\aleph_{0}$ and their weights are $\aleph_{1}$ because $\langle\mathbb{P}\rangle \simeq \omega$ Top $_{0}$ and $\langle\mathbb{B}\rangle \simeq 0$ Dim have precisely all countable coproducts. The first Kleene algebra has weight $\aleph_{0}$. PCAs built as syntactic models of the untyped $\lambda$-calculus typically have weight $\aleph_{0}$ because the corresponding sites have precisely all the finite coproducts.

Corollary 2.12 Let $\mathbb{A}$ be a $P C A$. In $\operatorname{Mod}(\mathbb{A})$, the coproduct of a non-trivial family $\left(X_{i}\right)_{i \in I}$ exists if, and only if, $|I|<\mathrm{w}(\mathbb{A})$.

Proof. The category $\langle\mathbb{A}\rangle$ is the full subcategory of $\operatorname{Mod}(\mathbb{A})$ on the canonically separated modest sets, which in turn is equivalent to the full subcategory $\operatorname{Proj}(\mathbb{A})$ on the regular projective modest sets over $\mathbb{A}$. It is easy to check that the inclusion of $\operatorname{Proj}(\mathbb{A})$ into $\operatorname{Mod}(\mathbb{A})$ preserves and reflects all coproducts that exist. By combining this with the fact that every modest set is covered by a regular epi whose domain is regular projective, we get the desired result.

As the Grothendieck topology on $\langle\mathbb{A}\rangle$ we take the coproduct topology $C$ which is generated by those families $\left\{f_{i}: Y_{i} \rightarrow X\right\}_{i \in I}$ for which $|I|<\mathrm{w}(\mathbb{A})$ and the morphism $\left[f_{i}\right]_{i \in I}: \coprod_{i \in I} Y_{i} \rightarrow X$ is an isomorphism.

Definition 2.13 The category of sheaves on $\langle\mathbb{A}\rangle$ for the coproduct topology is denoted by $\operatorname{Sh}(\mathbb{A})$.

Observe that the sheaves on $\langle\mathbb{A}\rangle$ are simply those presheaves $P$ that "preserve products", i.e., $P\left(\coprod_{i} Y_{i}\right) \cong \prod_{i} P Y_{i}$.

Theorem 2.14 The category $\operatorname{Sh}(\mathbb{A})$ is equivalent to the category of sheaves $\operatorname{Sh}(\operatorname{Mod}(\mathbb{A}), R)$ for the subcanonical Grothendieck topology $R$ generated by 
those families $\left\{f_{i}: B_{i} \rightarrow A\right\}_{i \in I}$ for which $|I|<\mathrm{w}(\mathbb{A})$ and the morphism $\left[f_{i}\right]_{i \in I}: \coprod_{i \in I} B_{i} \rightarrow A$ is a regular epi.

Proof. As in the proof of Corollary 2.12, we may replace $\langle\mathbb{A}\rangle$ with the equivalent category $\operatorname{Proj}(\mathbb{A})$ of regular projective modest sets over $\mathbb{A}$.

Let the jointly-split topology $S$ on $\operatorname{Proj}(\mathbb{A})$ be generated by those families $\left\{f_{i}: B_{i} \rightarrow A\right\}_{i \in I}$ for which $|I|<\mathrm{w}(\mathbb{A})$ and the morphism

$$
\left[f_{i}\right]_{i \in I}: \coprod_{i \in I} B_{i} \rightarrow A
$$

splits, i.e., has a right inverse $s: A \rightarrow \coprod_{i \in I} B_{i}$. Let us verify that the jointly split families form a basis for Grothendieck topology:

1. Isomorphisms cover: It is obvious that an isomorphism is covering since it is split by its inverse.

2. Stability under pullbacks: Suppose $\left\{f_{i}: B_{i} \rightarrow A\right\}_{i \in I}$ covers $A$. Consider the pullback along $g: C \rightarrow A$. Since coproducts in $\langle A\rangle$ are stable, we get a pullback diagram

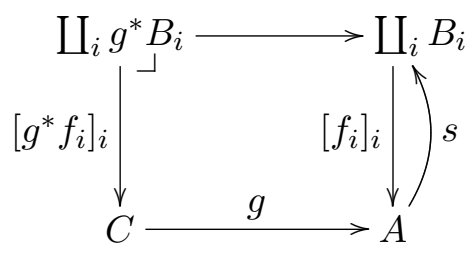

The morphism $s$ in the above diagram is the splitting of $\left[f_{i}\right]_{i}$. We want to show that the left-hand vertical morphism splits, which follows easily from the pullback property of the diagram. Since $\left[f_{i}\right]_{i} \circ s \circ g=$ $1_{C} \circ g$ there exists a unique arrow $t: C \rightarrow \coprod_{i} g^{*} B_{i}$ such that $1_{C}=$ $\left[g^{*} f_{i}\right]_{i} \circ t$, as required.

3. Transitivity: Suppose $\left\{f_{i}: B_{i} \rightarrow A\right\}_{i \in I}$ is a covering family, and for each $i \in I$, the family $\left\{g_{i j}: C_{i j} \rightarrow B_{i}\right\}_{j \in J_{i}}$ covers $B_{i}$. Then $\left[f_{i}\right]_{i}$ splits by a morphism $s$ and $\left[g_{i j}\right]_{j}$ splits by a morphism $r_{i}$, for each $i \in I$. The coproduct $C=\coprod_{i \in I} \coprod_{j \in J_{i}} C_{i j}$ exists because $\sum_{i \in I}\left|J_{i}\right|<\mathrm{w}(\mathbb{A})$. This is so because $\mathrm{w}(\mathbb{A})$ is a regular cardinal, $|I|<\mathrm{w}(\mathbb{A})$ and $\left|J_{i}\right|<\mathrm{w}(\mathbb{A})$ for all $i \in I$. The map $\left[f_{i} \circ g_{i j}\right]_{i j}: C \rightarrow A$ splits by $\left(\sum_{i \in I} r_{i}\right) \circ s$.

Next, we show that the jointly split families generate precisely the coproduct topology $C$. We need to show that a sieve $\left\{f_{i}: B_{i} \rightarrow A\right\}_{i \in I}$ contains an $S$ cover if, and only if, it contains a $C$-cover. One direction is easy, since every $C$-cover is obviously an $S$-cover. For the converse, if $\left\{f_{j}: B_{j} \rightarrow A\right\}_{j \in J}$ is jointly split by $s: A \rightarrow \coprod_{j \in J} B_{j}$, then we can decompose $A$ into a coproduct 
$A \cong \coprod_{j \in J} s^{*} B_{j}$, as in the pullback diagram

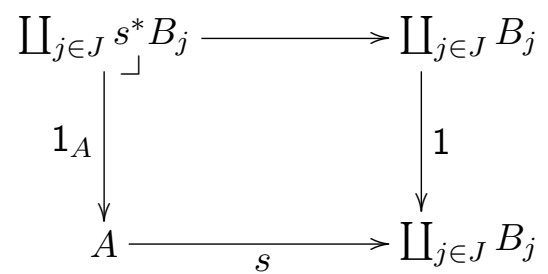

Therefore, if a sieve contains a jointly split family $\left\{f_{j}: B_{j} \rightarrow A\right\}_{j \in J}$, then it also contains a family whose coproduct is isomorphic to $A$.

As in the statement of the theorem, let $R$ be the Grothendieck topology on $\operatorname{Mod}(\mathbb{A})$ generated by those families $\left\{f_{i}: B_{i} \rightarrow A\right\}_{i \in I}$ for which $|I|<\mathrm{w}(\mathbb{A})$ and $\left[f_{i}\right]_{i \in I}: \coprod_{i \in I} B_{i} \rightarrow A$ is a regular epi. To finish the proof, we apply the Comparison Lemma $[M M 92$, Appendix, Corollary 4.3] to $\operatorname{Sh}(\operatorname{Mod}(\mathbb{A}), R)$ and $\operatorname{Sh}(\operatorname{Proj}(\mathbb{A}), S)$. For this we must check three conditions:

1. Topology $R$ is subcanonical: we chose $R$ to be generated by certain regular-epimorphic families.

2. Every object in $\operatorname{Mod}(\mathbb{A})$ is $R$-covered by objects in $\operatorname{Proj}(\mathbb{A})$ : this is equivalent to every modest set being a regular quotient of a regular projective modest set, which is the case.

3. A family $\left\{f_{i}: B_{i} \rightarrow A\right\}_{i \in I}$ is $S$-covering in $\operatorname{Proj}(\mathbb{A})$ if, and only if, it is $R$-covering in $\operatorname{Mod}(\mathbb{A})$ : this holds because a morphism $f: B \rightarrow A$ in $\operatorname{Proj}(\mathbb{A})$ is split if, and only if, it is a regular epi in $\operatorname{Mod}(\mathbb{A})$. Indeed, if it is split then it is a regular epi by a general category-theoretic argument. Conversely, suppose $f: B \rightarrow A$ is a regular epi and $A \in \operatorname{Proj}(\mathbb{A})$. Since $A$ is regular projective there exists a right inverse $s: B \rightarrow A$ of $f$, hence $f$ is split.

Corollary 2.15 The Yoneda embedding

$$
\operatorname{Mod}(\mathbb{A}) \stackrel{y}{\longrightarrow} \operatorname{Sh}(\mathbb{A})
$$

is full and faithful, preserves the locally cartesian closed structure, regular epis, and coproducts. In terms of categorical logic, it preserves and reflects validity of formulas involving full first-order logic, exponentials, dependent types, disjoint sum types, and quotients of $\neg \neg$-stable equivalence relations. In addition, $y$ preserves infinitary disjunctions and coproducts of cardinality less than $\mathrm{w}(\mathbb{A})$. In case $\mathrm{w}(\mathbb{A}) \geq \aleph_{1}, y$ also preserves the natural numbers object. 
More precisely, the functor $y$ is defined as follows. If $I:\langle\mathbb{A}\rangle \rightarrow \operatorname{Mod}(\mathbb{A})$ is the inclusion, then for $S \in \operatorname{Mod}(\mathbb{A}), y S=\operatorname{Hom}(I(\square), S)$ where the hom-set is taken in $\operatorname{Mod}(\mathbb{A})$. We do not have to compose with sheafification because the topology is subcanonical.

The Yoneda embedding $y$ itself extends similarly along the inclusion $J: \operatorname{Mod}(\mathbb{A}) \rightarrow \operatorname{RT}(\mathbb{A})$ of modest sets into the realizability topos for $\mathbb{A}$, to give a functor $Y: \operatorname{RT}(\mathbb{A}) \rightarrow \operatorname{Sh}(\mathbb{A})$, as indicated below.

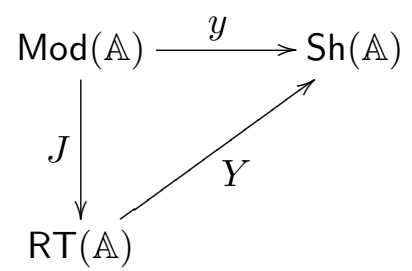

The functor $Y$ is defined much like $y$, i.e., for $X \in \mathrm{RT}(\mathbb{A})$, let $Y(X)=$ $\operatorname{Hom}(J(\square), X)$, where the hom-set is now taken in $\mathrm{RT}(\mathbb{A})$. It can be shown fairly easily that $Y$ also preserves first-order logic, but unlike $y$, it is not faithful (since the modest sets do not generate $\mathrm{RT}(\mathbb{A})$ ), nor does it preserve exponentials.

Example 2.16 Countably based equilogical spaces embed via the Yoneda embedding into the topos $\mathrm{Sh}(\mathbb{P}) \simeq \mathrm{Sh}\left(\omega \operatorname{Top}_{0}, C_{\omega}\right)$ of sheaves on the countably based $T_{0}$ spaces equipped with the countable coproducts topology.

Example 2.17 The topos $S h(\mathbb{K})$ is closely related to Mulry's recursive topos $\mathcal{R}$ [Mul82]. Specifically, a site for $\mathcal{R}$ is the category $\mathrm{R}$ of r.e. sets and partial recursive functions, equipped with the finite cover topology; the evident inclusion functor $i: \mathrm{R} \hookrightarrow\langle\mathbb{K}\rangle$ induces a geometric morphism $\operatorname{Sh}(\mathbb{K}) \rightarrow \mathcal{R}$ which, however, is not an equivalence. To see this, consider an immune set ${ }^{1} I \subseteq \mathbb{N}$ as an object of $\langle\mathbb{K}\rangle$. A non-recursive function $h: I \rightarrow I$ induces a natural endomorphism $h \circ-$ on the restricted representable functor $\operatorname{Hom}(i(-), I)$ in $\mathcal{R}$ which is not induced by any morphism in $\operatorname{Sh}(\mathbb{K})$.

Example 2.18 The category $\operatorname{Mod}(\mathbb{B})$ embeds into the topos $\mathrm{Sh}(\mathbb{B})$ which is equivalent to the topos $\mathrm{Sh}\left(0 \mathrm{Dim}, C_{\omega}\right)$, where $0 \mathrm{Dim}$ is the category of countably based 0-dimensional Hausdorff spaces.

\subsection{Functors Induced by Applicative Morphisms}

Recall that a discrete applicative morphism $\rho: \mathbb{E} \stackrel{\text { PCA }}{\longrightarrow} \mathbb{F}$ induces a functor $\widehat{\rho}: \operatorname{Mod}(\mathbb{E}) \rightarrow \operatorname{Mod}(\mathbb{F})$. This functor preserves finite limits and regular epis by [Lon94]. Suppose that in addition $\widehat{\rho}$ preserves those coproducts

\footnotetext{
${ }^{1}$ Recall that an immune set is an infinite set which does not contain any infinite r.e. sets, see e.g. [Rog87].
} 
that $\operatorname{Mod}(\mathbb{E})$ has, and call such a functor +-preserving. Then $\widehat{\rho}$ preserves covering families, and so (by [MM92, Section VII.7]) induces a geometric morphism $\left(\rho^{*}, \rho_{*}\right): \operatorname{Sh}(\mathbb{F}) \rightarrow \operatorname{Sh}(\mathbb{E})$ between the corresponding sheaf toposes, as in the diagram below.

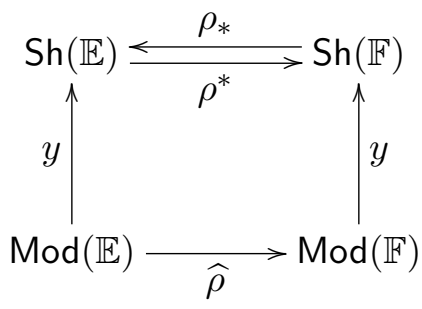

The inverse image part $\rho^{*}$ of the geometric morphism in the above diagram makes the evident square commute up to natural isomorphism.

\subsection{Applicative Retractions Induce Local Maps of Toposes}

A pair of applicative morphisms $\delta: \mathbb{E} \stackrel{\text { PCA }}{\longrightarrow} \mathbb{F}$ and $\eta: \mathbb{F} \stackrel{\text { PCA }}{\longrightarrow} \mathbb{E}$ such that $1_{\mathbb{F}} \preceq \delta \circ \eta$ and $\eta \circ \delta \preceq 1_{\mathbb{E}}$ is called an applicative adjunction, written $(\eta \dashv$ $\delta): \mathbb{E} \stackrel{\text { PCA }}{\longrightarrow} \mathbb{F}$, see [Lon94]. An applicative adjunction for which $\delta \circ \eta \sim 1_{\mathbb{F}}$ is called an applicative retraction.

Example 2.19 As Peter Lietz observed, the morphisms $(\iota \dashv \delta): \mathbb{P} \stackrel{\mathrm{PCA}}{\longrightarrow} \mathbb{B}$ of example 2.6 form an applicative retraction. See [Bau00] for details.

Let $(\eta \dashv \delta): \mathbb{E} \stackrel{\text { PCA }}{\longrightarrow} \mathbb{F}$ be an applicative retraction of discrete applicative morphisms. Then the induced functors $\widehat{\eta}$ and $\widehat{\delta}$ form an adjoint pair $\widehat{\eta} \dashv \widehat{\delta}$,

$$
\operatorname{Mod}(\mathbb{E}) \underset{\widehat{\delta}}{\stackrel{\widehat{\eta}}{\longleftarrow}} \operatorname{Mod}(\mathbb{F})
$$

In addition, $\widehat{\delta} \circ \widehat{\eta} \cong 1_{\operatorname{Mod}(\mathbb{F})}$. Suppose that $\widehat{\delta}$ is + -preserving. Combining this with (1), we get three adjoint functors $\eta^{*} \dashv \eta_{*}=\delta^{*} \dashv \delta_{*}$,

$$
\operatorname{Sh}(\mathbb{E}) \underset{\eta_{*}=\delta^{*}}{\stackrel{\eta^{*}}{\delta_{*}}} \mathrm{Sh}(\mathbb{F})
$$

where $\delta^{*} \circ \eta^{*} \cong 1_{\mathrm{Sh}(\mathbb{F})}$. Thus, a +-preserving discrete applicative retraction $(\eta \dashv \delta): \mathbb{E} \stackrel{\text { PCA }}{\longrightarrow} \mathbb{F}$ induces a local map of toposes $\operatorname{Sh}(\mathbb{E}) \rightarrow \operatorname{Sh}(\mathbb{F})$. Such maps were studied by Birkedal in [Bir99]; there and in [ABS99] a $\sharp-b$ calculus for the internal logic is obtained, which can be used here to compare realizability in modest sets over $\mathbb{E}$ with that over $\mathbb{F}$. 
An applicative retraction does not seem to induce a third adjoint if we use realizability toposes $\mathrm{RT}(\mathbb{E})$ and $\mathrm{RT}(\mathbb{F})$ instead of sheaf toposes (although one might consider relative realizability [Bir99, ABS99]). On the other hand, many applicative retractions that we have encountered are +-preserving, and so they induce local maps of sheaf toposes. A good conceptual explanation of this phenomenon would be desirable.

\subsection{A Forcing Semantics for Realizability}

The following theorem spells out the Kripke-Joyal semantics in $\operatorname{Sh}(\mathbb{A})$, see [MM92, Section VI.6]. The interpretation of disjunction, negation and existential quantification is simpler than the usual one due to the simple nature of the coproduct topology on the site $\langle\mathbb{A}\rangle$.

Theorem 2.20 Let $X, Y \in \operatorname{Sh}(\mathbb{A})$, and let

$$
x: X|\phi(x) \quad x: X| \psi(x) \quad x: X, y: Y \mid \rho(x, y)
$$

be formulas in the internal language of $\operatorname{Sh}(\mathbb{A})$. Let $A \in\langle\mathbb{A}\rangle$ and $a \in X A$. The Kripke-Joyal forcing relation $\models$ is interpreted as follows:

1. $A \models \phi(a) \wedge \psi(a)$ if, and only if, $A \models \phi(a)$ and $A \models \psi(a)$.

2. $A \models \phi(a) \vee \psi(a)$ if, and only if, there exist $A_{1}, A_{2} \in\langle\mathbb{A}\rangle$ such that $A=A_{1}+A_{2}$, with $A_{1} \models \phi\left(a \cdot \iota_{1}\right)$ and $A_{2} \models \psi\left(a \cdot \iota_{2}\right)$.

3. $A=\phi(a) \longrightarrow \psi(a)$ if, and only if, for all $f: B \rightarrow A$ in $\langle\mathbb{A}\rangle$, if $B=\phi(a \cdot f)$ then $B=\psi(a \cdot f)$.

4. $A \models \neg \phi(a)$ if, and only if, for all $f: B \rightarrow A$ in $\langle\mathbb{A}\rangle$, if $B \models \phi(a \cdot f)$ then $B=0$.

5. $A=\forall y \in Y . \rho(a, y)$ if, and only if, for all $f: B \rightarrow A$ in $\langle\mathbb{A}\rangle$ and all $b \in Y B$, one has $B \models \rho(a \cdot f, b)$.

6. $A=\exists y \in Y . \rho(a, y)$ if, and only if, $A=\coprod_{i \in I} A_{i}$ in $\langle\mathbb{A}\rangle,|I|<\mathrm{w}(\mathbb{A})$, and for each $i \in I$ there exist $b_{i} \in Y A_{i}$ such that $A_{i}=\rho\left(a \cdot \iota_{i}, b_{i}\right)$.

Proof. We only need to show that the standard interpretations of disjunction, negation, and existential quantification simplify to the forms stated in the theorem. This follows easily from the characterization of the topology via the disjoint sum basis.

Let us first consider disjunction. Suppose $A=\phi(a) \vee \psi(a)$. Then there exists a family $\left\{\iota_{i}: A_{i} \rightarrow A\right\}_{i \in I}$ such that $\left[\iota_{i}\right]_{i}: A_{1}+\cdots+A_{k} \rightarrow A$ is an isomorphism and, for every $i \in I, A_{i} \models \phi\left(a \cdot \iota_{i}\right)$ or $A_{i} \models \psi\left(a \cdot \iota_{i}\right)$. Define the sets $J$ and $K$ by

$$
J=\left\{i \in I \mid \models \phi\left(a \cdot \iota_{i}\right)\right\}, \quad K=I \backslash J .
$$


Let $A_{1}^{\prime}=\coprod_{j \in J} A_{j}$ and $A_{2}^{\prime}=\coprod_{k \in K} A_{k}$. Then it is clear that $A=A_{1}^{\prime}+A_{2}^{\prime}$. Let $\kappa_{1}=\left[\iota_{j}\right]_{j \in J}: A_{1} \rightarrow A$ and $\kappa_{2}=\left[\iota_{k}\right]_{k \in K}: A_{2} \rightarrow A$ be the isomorphisms. It is now clear that $A_{1}^{\prime}=\phi\left(a \cdot \kappa_{1}\right)$ and $A_{2} \mid=\psi\left(a \cdot \kappa_{2}\right)$, as required. The converse holds, since if $A=A_{1}+A_{2}, A_{1}=\phi\left(a \cdot \iota_{1}\right)$ and $A_{2}=\psi\left(a \cdot \iota_{2}\right)$, then $A \models \phi(a) \vee \psi(a)$ because the sum of canonical inclusions $\left[\iota_{1}, \iota_{2}\right]: A_{1}+A_{2} \rightarrow A$ is an isomorphism, thus it covers $A$.

The interpretation of negation is correct because an object is covered by the empty family \{\} if, and only if, it is the initial object 0 .

Suppose $A=\exists y \in Y . \rho(a, y)$. Then there is a family $\left\{\iota_{i}: A_{i} \rightarrow A\right\}_{i \in I}$ such that $A=\coprod_{i \in I} A_{k}, \iota_{i}: A_{i} \rightarrow A$ is the canonical inclusion for every $i \in I$, and there exists $b_{i} \in Y A_{i}$ such that $A_{i} \mid=\rho\left(a \cdot \iota_{i}, b_{i}\right)$. This proves one direction. The converse is proved easily as well.

If $\operatorname{Mod}(\mathbb{A})$ has countable coproducts a clause involving countable disjunctions can be added. The forcing semantics can be restricted to the modest sets, as long as the formulas are restricted to first-order logic with exponentials, dependent types, subset types, and quotients of $\neg \neg$-stable equivalence relations. It is a consequence of Corollary 2.15 that such a formula is valid in the forcing semantics above if, and only if, it is valid in the realizability interpretation.

\subsection{A Transfer Principle for Modest Sets}

Suppose $(\eta \dashv \delta): \mathbb{E} \stackrel{\text { PCA }}{\longrightarrow} \mathbb{F}$ is an applicative retraction such that $\widehat{\delta}$ is +preserving. The transfer principle from Awodey et. al. [ABS99] can be applied to the induced local map of toposes,

$$
\operatorname{Sh}(\mathbb{E}) \underset{\eta_{*}=\delta^{*}}{\stackrel{\eta_{*}^{*}}{\longrightarrow}} \operatorname{Sh}(\mathbb{F}) .
$$

We say that a formula $\theta$ in the internal language of a topos is $l o c a l^{2}$ if it is built from atomic predicates, including equations, using first-order logic, and if in every subformula of the form $\phi \longrightarrow \psi$, there is no $\forall$ or $\longrightarrow$ in $\phi$.

If $\theta$ is a local sentence in the internal logic of $\operatorname{Sh}(\mathbb{F})$, we write $\operatorname{Sh}(\mathbb{F}) \models \theta$ when the canonical interpretation of $\theta$ is valid in $\operatorname{Sh}(\mathbb{F})$. The sentence $\theta$ can also be interpreted in $\operatorname{Sh}(\mathbb{E})$ by mapping the types and relations occurring in $\theta$ over to $\operatorname{Sh}(\mathbb{E})$ with $\eta^{*}$. The transfer principle from [ABS99] then tells us that for such a local sentence $\theta$,

$$
\operatorname{Sh}(\mathbb{E}) \models \theta \quad \text { if and only if } \quad \operatorname{Sh}(\mathbb{F}) \models \theta .
$$

It follows that if only types and relations from $\operatorname{Mod}(\mathbb{F})$ occur in $\theta$ then the transfer principle restricts to the categories of modest sets:

$$
\operatorname{Mod}(\mathbb{E}) \models \theta \quad \text { if and only if } \quad \operatorname{Mod}(\mathbb{F}) \models \theta .
$$

\footnotetext{
${ }^{2}$ In [ABS99] such a formula is called "stable".
} 
Here we interpret $\theta$ in $\operatorname{Mod}(\mathbb{E})$ by mapping all types and relations that occur in $\theta$ over to $\operatorname{Mod}(\mathbb{E})$ by $\widehat{\eta}$. The notation $\operatorname{Mod}(\mathbb{E}) \models \theta$ means that the sentence $\theta$ is valid in the standard realizability interpretation, or equivalently, in the forcing semantics as described in Theorem 2.20. The following theorem explains why $\operatorname{Mod}(\mathbb{P})$ and $\operatorname{Mod}(\mathbb{B})$ appear to be very similar, at least as far as simple types are concerned.

Theorem 2.21 Let $(\iota \dashv \delta): \mathbb{P} \stackrel{\mathrm{PCA}}{\longrightarrow} \mathbb{B}$ be the applicative retraction from Example 2.19. Let $\theta$ be a local sentence such that all variables occurring in $\theta$ have types $\mathbb{N}, \mathbb{N}^{\mathbb{N}}$, or $\mathbb{R}$. Then

$$
\operatorname{Mod}(\mathbb{P})=\theta \quad \text { if and only if } \quad \operatorname{Mod}(\mathbb{B}) \models \theta,
$$

where $\mathbb{N}$ is interpreted as the natural numbers object, $\mathbb{N}^{\mathbb{N}}$ is interpreted as the obvious exponential, and $\mathbb{R}$ is interpreted as the object of Cauchy real numbers.

Proof. The theorem holds because the functor $\widehat{\iota}: \operatorname{Mod}(\mathbb{B}) \rightarrow \operatorname{Mod}(\mathbb{P})$ preserves the natural numbers object $\mathbb{N}$, its function space $\mathbb{N}^{\mathbb{N}}$, and the real numbers object $\mathbb{R}$. It is not hard to see that $\widehat{\delta}$ preserves countable coproducts, which are precisely the coproducts that $\operatorname{Mod}(\mathbb{P})$ has.

In Theorem 2.21 we cannot allow variables of higher types such as $\mathbb{N}^{\mathbb{N}}$ and $\mathbb{R}^{\mathbb{R}}$ to occur, because it is well known that the following local sentence involving $\mathbb{N}^{\mathbb{N}}$ is valid in $\operatorname{Mod}(\mathbb{B})$ but not in $\operatorname{Mod}(\mathbb{P})$ :

$$
\forall F \in \mathbb{N}^{\mathbb{N}^{\mathbb{N}}} \cdot \exists \alpha \in \mathbb{N}^{\mathbb{N}} \cdot \forall \beta \in \mathbb{N}^{\mathbb{N}} \cdot F \beta=(\alpha \mid \beta) .
$$

The sentence states that every functional $F \in \mathbb{N}^{\mathbb{N}}$ has an associate $\alpha \in \mathbb{N}^{\mathbb{N}}$ in the sense of Kleene [Kle59]. Here $\alpha \mid \beta$ is Kleene's continuous function application. The statement $n=(\alpha \mid \beta)$ is equivalent to

$$
\exists m \in \mathbb{N} .(\alpha(\bar{\beta} m)=n+1 \wedge \forall k \in \mathbb{N} .(k<m \longrightarrow \alpha(\bar{\beta} k)=0)) .
$$

Similarly, a statement that all functions $f \in \mathbb{R}^{\mathbb{R}}$ are continuous is valid in $\operatorname{Mod}(\mathbb{B})$ but not in $\operatorname{Mod}(\mathbb{P})$. Thus, in a roundabout way, we obtain the following result.

Corollary 2.22 The functor $\widehat{\iota}: \operatorname{Mod}(\mathbb{B}) \rightarrow \operatorname{Mod}(\mathbb{P})$ does not preserve exponentials. In particular, $\widehat{\iota}\left(\mathbb{N}^{\mathbb{N}}\right)$ is not isomorphic to the object $\mathbb{N}^{\mathbb{N}^{\mathbb{N}}}$ in $\operatorname{Mod}(\mathbb{P})$, and $\widehat{\iota}\left(\mathbb{R}^{\mathbb{R}}\right)$ is not isomorphic to the object $\mathbb{R}^{\mathbb{R}}$ in $\operatorname{Mod}(\mathbb{P})$.

We can in fact prove Corollary 2.22 directly as follows. Let $X=\mathbb{N}^{\mathbb{N}^{\mathbb{N}}}$ be the object of type 2 functionals in $0 \mathrm{Equ}$, which is equivalent to $\operatorname{Mod}(\mathbb{B})$, and let $Y=\mathbb{N}^{\mathbb{N}}$ be the object of type 2 functionals in Equ. Both $X$ and $Y$ are equilogical spaces. The space $|X|$ is a Hausdorff space. The space $|Y|$ is the 
subspace of the total elements of the Scott domain $D=\left[\mathbb{N}_{\perp}{ }^{\omega} \rightarrow \mathbb{N}_{\perp}\right]$. The equivalence relation on $|Y|$ is the consistency relation of $D$ restricted to $|Y|$. Suppose $f:|Y| \rightarrow|X|$ represented an isomorphism, and let $g:|X| \rightarrow|Y|$ represent its inverse. Because $f$ is monotone in the specialization order and $|X|$ has a trivial specialization order, $a \equiv_{Y} b$ implies $f x=f y$. Therefore, $g \circ f:|Y| \rightarrow|Y|$ is an equivariant retraction. By [Bau00, Proposition 4.1.8], $Y$ is a topological object. By [Bau00, Corollary 4.1.9], this would mean that the topological quotient $|Y| / \equiv_{Y}$ is countably based, but it is not, as is well known.

Another way to see that $Y$ cannot be topological is to observe that $Y$ is an exponential of the Baire space, but the Baire space is not exponentiable in $\omega$ Top $_{0}$, and in particular $\mathbb{N}^{\mathbb{N}^{\mathbb{N}}}$ is not a topological object.

Finally, we remark that statement $(2)$ is of course valid in $\operatorname{Mod}(\mathbb{P})$ if $\mathbb{N}^{\mathbb{N}}$ is replaced by $\widehat{\iota}\left(\mathbb{N}^{\mathbb{N}}\right)$. But then it becomes a simple truism, since it can be shown that in $\operatorname{Mod}(\mathbb{P})$ the space $\widehat{\iota}\left(\mathbb{N}^{\mathbb{N}}\right)$ is just the set of those functionals that have an associate:

$$
\widehat{\iota}\left(\mathbb{N}^{\mathbb{N}}\right)=\left\{F \in \mathbb{N}^{\mathbb{N}} \mid \exists \alpha \in \mathbb{N}^{\mathbb{N}} \cdot \forall \beta \in \mathbb{N}^{\mathbb{N}} \cdot F \beta=(\alpha \mid \beta)\right\} .
$$

Corollary 2.22 should be contrasted with the fact that there is an epimono $\widehat{\iota}\left(\mathbb{N}^{\mathbb{N}^{\mathbb{N}}}\right) \rightarrow \mathbb{N}^{\mathbb{N}^{\mathbb{N}}}$ that is not iso but nevertheless induces a natural bijection between the global points of $\widehat{\iota}\left(\mathbb{N}^{\mathbb{N}}\right)$ and the global points of $\mathbb{N}^{\mathbb{N}}$. In this sense, the finite types over $\mathbb{N}$ in $\operatorname{Mod}(\mathbb{P})$ and $\operatorname{Mod}(\mathbb{B})$ are equivalent as far as the cartesian closed structure is concerned, but here we see that they have different logical properties.

\section{References}

[ABS99] S. Awodey, L. Birkedal, and D.S. Scott. Local realizability toposes and a modal logic for computability. In L. Birkedal, J. van Oosten, G. Rosolini, and D.S. Scott, editors, Tutorial Workshop on Realizability Semantics, FLoC'99, Trento, Italy, 1999, volume 23 of Electronic Notes in Theoretical Computer Science. Elsevier, 1999. To appear in Math. Stru. Comp. Sci.

[Bau00] A. Bauer. The Realizability Approach to Computable Analysis and Topology. PhD thesis, Carnegie Mellon University, 2000. Available as CMU technical report CMU-CS-00-164 and at http://andrej . $\mathrm{com} /$ thesis.

[BBS98] A. Bauer, L. Birkedal, and D.S. Scott. Equilogical spaces. Preprint submitted to Elsevier, 1998.

[Bir99] L. Birkedal. Developing Theories of Types and Computability. PhD thesis, School of Computer Science, Carnegie Mellon University, 
December 1999. Available as CMU Technical Report: CMU-CS99-173.

[Bir00] L. Birkedal. Developing theories of types and computability via realizability. Electronic Notes in Theoretical Computer Science, 34, 2000. Available at http://www.elsevier.nl/locate/entcs/volume34.html.

[Jec97] T. Jech. Set Theory, Second Edition. Springer, 1997.

[JM89] P.T. Johnstone and I. Moerdijk. Local maps of toposes. Proc. London Math. Soc., 3(58):281-305, 1989.

[Kle59] S.C. Kleene. Countable functionals. In Constructivity in Mathematics, pages 81-100, 1959.

[KV65] S.C. Kleene and R.E. Vesley. The Foundations of Intuitionistic Mathematics, especially in relation to recursive functions. NorthHolland Publishing Company, 1965.

[Lon94] J.R. Longley. Realizability Toposes and Language Semantics. PhD thesis, University of Edinburgh, 1994.

[MM92] S. Mac Lane and I. Moerdijk. Sheaves in Geometry and Logic. A First Introduction to Topos Theory. Springer-Verlag, New York, 1992.

[Mul82] P. Mulry. Generalized Banach-Mazur functionals in the topos of recursive sets. Journal of Pure and Applied Algebra, 26:71-83, 1982.

[Rog87] H. Rogers. Theory of Recursive Functions and Effective Computability. The MIT Press, 1987.

[Ros86] G. Rosolini. Continuity and Effectiveness in Topoi. PhD thesis, University of Oxford, 1986.

[RS99] P. Rosolini and Th. Streicher. Comparing models of higher type computation. In L. Birkedal, J. van Oosten, G. Rosolini, and D.S. Scott, editors, Tutorial Workshop on Realizability Semantics, FLoC'99, Trento, Italy, 1999, volume 23 of Electronic Notes in Theoretical Computer Science. Elsevier, 1999.

[Sco76] D.S. Scott. Data types as lattices. SIAM Journal of Computing, 5(3):522-587, 1976.

[Wei00] Klaus Weihrauch. An Introduction to Computable Analysis. Springer, Berlin, 2000. 